\title{
Étude, par photographie aérienne numérisée, de la variabilité intraparcellaire d'une culture de soja diversement irriguée
}

\author{
F Levrault *, J Puech \\ Participation technique : J Salvy \\ Centre de Recherches INRA, Toulouse-Auzeville, station d'agronomie, chemin de Borde-Rouge BP 27, \\ 31326 Castanet-Tolosan Cedex, France
}

(Reçu le 2 août 1991; accepté le 23 novembre 1992)

\begin{abstract}
Résumé - Dans l'optique d'une gestion plus performante de l'eau dans les systèmes de culture, l'utilisation de la photographie aérienne infrarouge couleur (IRC) associée au traitement d'image informatisé est testée sur une culture de soja (Glycine max (L) Merr). Les résultats montrent que cet outil permet une appréhension spatio-temporelle fiable de l'alimentation en eau des plantes. Les réponses enregistrées dans le canal rouge des photographies numérisées, sont corrélées $\left(r^{2}=0,71\right.$ à 0,87$)$ avec des paramètres liés à l'alimentation des plantes en eau : indice foliaire, matière sèche totale, hauteur d'eau apportée. Deux de ces relations sont utilisées dans leur domaine de validité pour calculer différentes cartes thématiques relatives au développement (indice foliaire) ou au traitement (lame d'eau). Ces documents, permettent la prise en compte de l'hétérogénéité des sols, l'estimation des niveaux de développement en tout point de la parcelle et le contrôle des régimes hydriques. Ils constituent des outils spatiaux d'aide à la décision. L'amélioration et les possibilités de généralisation des résultats sont envisagées.
\end{abstract}

gestion eau / télédétection / soja

Summary - Digitized aerial photography study of intraparcel variation in a soybean plot with different degrees of irrigation. Aerial infra-red color photography and computer image processing have been tested in an experimental soybean plot survey in order to improve water management in cropping systems. Results show that a new approach to water requirements can be adopted over time and with respect to spatial dimension. Good agreements $\left(\mathrm{r}^{2}=\right.$ 0.71-0.87) were obtained between digital responses of digitized photographs (red channel) and plant water status indicators (leaf area index, total dry biomass, water amount). These relations were used to convert digitized photographs into different thematic maps (relative to development and treatment levels). These maps allowed variability to be taken into account and provided developmental level assessment throughout the plot. They can be used for water treatment control and provide data for decision making. Improvement and the possibilities for generalized application of these results are discussed.

water management / remote sensing / soybean

\section{INTRODUCTION}

Dans un contexte d'optimisation de la production agricole, les impératifs biologiques, techniques, économiques et écologiques doivent être conciliés. À la station d'agronomie de l'INRA de Toulouse-Auzeville, le dispositif expérimental d'étude des rotations culturales et des niveaux d'intensification permet de préciser le rôle de ces différentes contraintes dans le cadre de l'optimisation technico-économique d'assolements de cultures à graines (Marty et al, 1984). Parmi les nombreux facteurs analysés (rotations culturales, travail du sol, fertilisation, alimentation hydrique, traitements phytosanitaires), l'eau représente un élément prépondérant d'accroissement des rendements, mais également des charges.

\footnotetext{
* Correspondance et tirés à part
} 
Une gestion de l'eau performante est de plus en plus nécessaire à la bonne marche économique des exploitations agricoles. À l'échelle régionale, ce besoin est exacerbé par l'extension des cultures d'été et par les problèmes de limitation des ressources en eau et de pollution des nappes par les nitrates.

Sur les dispositifs expérimentaux, les études concernant la gestion de l'eau font généralement appel à des indicateurs des besoins en eau des plantes. Les indicateurs les plus couramment employés (données climatiques, phénologie, humidimétrie neutronique, tensiométrie, potentiel foliaire, micromorphométrie, etc) présentent $2 \mathrm{li}$ mites majeures : une lourdeur d'emploi (coût, temps de mesure) et/ou une faible représentativité spatiale qui permettent difficilement une approche spatio-temporelle du thème hydrique. En matière de gestion de l'eau des progrès paraîssent donc réalisables, à condition de disposer d'un indicateur spatial des besoins en eau des plantes.

Compte tenu de ses caractéristiques intrinsèques, l'imagerie visible et proche infrarouge répond à ce besoin, tout d'abord en raison des relations existant entre la réponse spectrale et plusieurs descripteurs agronomiquement pertinents de l'état hydrique du peuplement végétal (Tucker et al, 1979; Holben et al, 1980; Kolenkark et al, 1982), ensuite, en raison du caractère non destructif et de la rapidité des mesures, qui en permettent la répétitivité. Enfin, parce que l'acquisition synoptique des données permet la description exhaustive de surfaces. Elle doit cependant être disponible sous une forme compatible avec le suivi de parcelles de moins de 1 ha, ce qui exclut actuellement le recours à l'imagerie satellitaire. D'où l'intérêt de la photographie aérienne infrarouge couleurs (IRC), qui délivre des données spectrales avec une résolution spatiale et une répétitivité adaptées au suivi d'un dispositif expérimental (Curran, 1985; Long et al, 1989). De plus, le développement de logiciels de numérisation et de traitement d'image permet à présent une analyse automatisée des informations spectrales contenues dans les prises de vue (Everitt et al, 1986).

En utilisant ces atouts, nous avons voulu montrer l'intérêt de la photographie IRC associée au traitement d'image, en matière de gestion de l'eau dans le cadre de la conduite culturale. Toutefois, sur le système de culture décrit précédemment, la multiplicité des facteurs en jeu rend l'interprétation des réponses spectrales délicate, lors d'une première approche. Aussi, par souci de clarification, nous avons choisi, pour cette étude, d'évaluer l'outil sur un site test, avec l'eau comme unique facteur de différenciation mis en jeu. Pour ce faire, nous avons analysé par imagerie visible et proche infrarouge plusieurs parcelles de soja soumises à différents niveaux d'intensification hydrique afin :

- d'abord, d'établir des liaisons significatives entre les réponses spectrales enregistrées et différents descripteurs de terrain liés à l'alimentation des plantes en eau;

- ensuite, de cartographier ces descripteurs (les quantifier et détailler leur répartition spatiale) à différentes dates sur la totalité du dispositif observé, à partir des liaisons précédemment établies;

- enfin, d'illustrer, au travers d'un exemple, les conséquences d'une telle hétérogénéité sur les prises de décisions relatives à la gestion de l'eau sur la parcelle;

\section{MATÉRIEL ET MÉTHODES}

L'étude est réalisée en 1990 sur une parcelle de soja (Glycine max (L) Merr) de $82 \times 42 \mathrm{~m}$. Le sol (terrefort du Lauragais) est profond, argilo-limoneux, légèrement calcaire. Le semis a été réalisé le 2 mai (sens NW-SE, densité 450000 pieds/ha, écartement $50 \mathrm{~cm}$ ). La levée a eu lieu le 13 mai.

Quatre traitements hydriques sont mis en place, sur 4 parcelles (nos 1-4) recevant des hauteurs totales d'eau comprises entre 50 et $300 \mathrm{~mm}$ (figure $\mathrm{n}^{\circ} 1$ ). Pour tous les traitements, 6 apports ( 1 tous les 10 j) sont réalisés entre le $45^{\mathrm{e}}$ et le $102^{\mathrm{e}} \mathrm{j}$ après la levée, correspondant à la phase de sensibilité du soja au stress hydrique (Mingeau, 1974). On obtient ainsi une large gamme de couverture des besoins en eau des plantes (rendements en grain entre 10 et 35 ha). Pour chaque traitement, les apports sont réalisés par un asperseur décrivant un demi-cercle (surface $250 \mathrm{~m}^{2}$, rayon $9 \mathrm{~m}$ ), afin d'obtenir un gradient de répartition de l'eau. Les hauteurs d'eau sont mesurées au moyen d'un pluviomètre à lecture directe placé a mi-portée de l'asperseur. Le gradient des apports est contrôlé par 9 pluviomètres d'appoint, placés tous les mètres le long de l'axe de symétrie de chaque parcelle. Durant la période des irrigations, la hauteur de pluie a été de $80 \mathrm{~mm}$.

Deux missions aériennes ont été effectuées les 25 juin et 27 août ( 44 et 103 j après la levée). Les prises de vue (vitesse $1 / 1500 \mathrm{~s}, \mathrm{f} 5,6$ ) sont réalisées verticalement par temps clair, sans vent, entre $11 \mathrm{~h} 30$ et $12 \mathrm{~h} 30 \mathrm{TU}$, depuis un ultra léger motorisé volant à $400 \mathrm{~m}$ d'altitude et à la vitesse de $60 \mathrm{~km} / \mathrm{h}$. II a été utilisé le film infrarouge couleurs Kodak Aerochrome 2443, 500 ASA. L'appareil photographique (Hasselblad format $5,7 \times 5,7 \mathrm{~cm}$ ) est équipé d'un objectif de $80 \mathrm{~mm}$ de distance focale et d'un filtre jaune (Kodak 


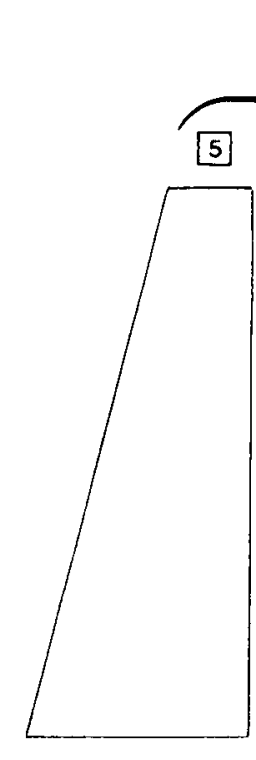

partie utilisée pour les prélèvements complémentaires

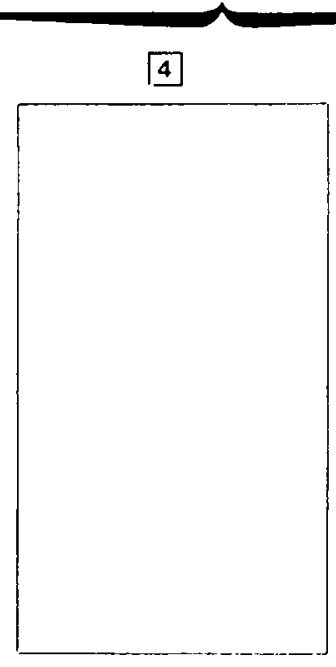

3

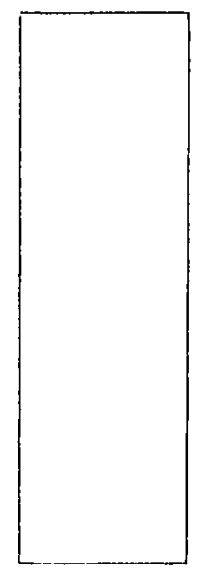

0 partie utilisée par les quatres régimes hydriques

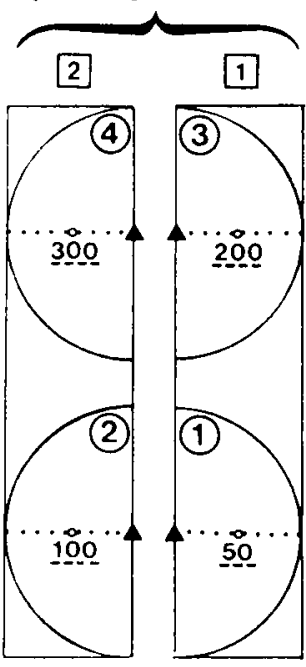

Legende:

1 : numero du bloc
(1) : numéro de la parcelle
: portec des arroseurs

100 : lame d'eau apporté au niveau du pluviometre de contróle

$\Delta$ : asperseur

0 : pluviomètre de contrôle

- : pluviomètre d'appoint o

Fig 1. Dispositif expérimental.

Wratten $n^{\circ}$ 12) destiné à stopper les rayonnements bleu et UV.

Après chaque mission, 36 prélèvements de $1,5 \mathrm{~m}^{2}$ ( $1 \mathrm{~m} \times 1,5 \mathrm{~m})$ sont effectués dans des parties différemment développées, consécutives à l'hétérogénéité des sols et/ou des apports ( 3 par parcelle et 24 sur le reste du dispositif). Sur ces prélèvements sont mesurés, l'indice foliaire (par planimétrie optique) puis la matière sèche totale (après passage à l'étuve : $60^{\circ} \mathrm{C}$ pendant $48 \mathrm{~h})$.

L'analyse des photographies IRC est réalisée au moyen du progiciel de traitement d'image CARTO-PC (école des Mines de Paris). La numérisation des diapositives est faite au moyen d'une caméra noir et blanc haute résolution (Cohu ref 4710 Solid State), les diapositives étant placées sur une table lumineuse (Macbeth Prooflite 516). Les valeurs numériques obtenues sur les images (liées aux densités optiques des points correspondants sur le film) ne sont pas calibrées en valeurs de réflectance, mais on cherche à ce que ces valeurs soient comparables entre elles. Pour cela, il faut s'affranchir de l'influence de l'angle zénithal de visée, de l'effet de vignettage de l'optique, de l'hétérogénéité éventuelle du développement du film, et de l'hétérogénéité d'éclairement de la table lumineuse. Dans ce but, nous avons limité l'utilisation de chaque photographie à la partie centrale correspondant aux angles zénithaux de visée inférieurs à 10 degrés (soit $20 \%$ de la surface de chaque photographie). La résolution spatiale des images numériques est de $14 \mathrm{~cm}$ en ligne et de $22 \mathrm{~cm}$ en colonne si bien que les 3245 $\mathrm{m}^{2}$ de la culture sont décrits par 105364 pixels. Les liaisons entre les descripteurs de terrain et la réponse spectrales sont établies empiriquement en calculant, sur les images numériques, la réponse moyenne des 50 pixels correspondant à chaque zone de prélèvement (module de calcul statistique du progiciel). Les cartes de répartition des descripteurs de terrain sont réalisées en convertissant les images numériques initiales au moyen des liaisons précédentes (module de combinaison arithmétique du progiciel).

Compte tenu des dates des 2 missions, le canal proche infrarouge n'amène pas d'information supplémentaire par rapport au canal rouge en raison (1) de la faible couverture du sol observée à la première mission et (2) de l'avancement des processus de sénescence lors de la deuxième mission. Dans ces conditions, les résultats présentés ici sont issus uniquement du canal rouge des photographies IRC. Le film IRC restituant les longueurs d'onde du vert, du rouge et du proche infrarouge par les couleurs bleue verte et rouge respectivement, il est donc utilisé un filtre vert (Kodak Wratten réf 58 ) pour extraire le canal rouge des prises de vue.

\section{RÉSULTATS}

\section{Liaisons entre descripteurs de terrain et réponse spectrale}

Des relations fiables sont obtenues entre les réponses enregistrées de l'indice foliaire, la matière sèche totale ou la hauteur d'eau apportée. 


\section{Indice foliaire}

La figure 2 montre la liaison observée le 25 juin 1990 entre l'indice foliaire des 36 prélèvements et les réponses correspondantes sur l'image numérique. Malgré l'utilisation de données spectrales non calibrées, on observe une relation asymptotique décroissante $(y=152,9(1 / x)-$ 0,19 ) de même type qu'en radiométrie en raison des conditions similaires d'acquisition des valeurs numériques pour les différents points de l'image. La qualité de la relation $\left(r^{2}=0,71\right)$ démontre que le canal rouge autorise, à cette date, une différenciation fiable des niveaux d'indice foliaire. On note toutefois une saturation logique des valeurs numériques pour les indices foliaires $>4$, soulignant l'intérêt du canal proche infrarouge pour différencier les biomasses supérieures. À cette date, les irrigations n'ayant pas encore été mises en place, la large gamme d'indices foliaires observées (de 0,9 à 5,3 ) souligne l'influence des hétérogénéités de sol sur le développement de la végétation.

\section{Matière sèche totale}

La figure $\mathrm{n}^{\circ} 3$ détaille la relation observée le 25 juin 1990 entre la matière sèche totale par unité de surface des 36 points de prélèvement et les réponses correspondantes sur l'image numérique. Comme précédemment, on observe une relation asymptotique décroissante : $y=9798,2$ $\left.(1 / x)-19,25 ; r^{2}=0,71\right)$. Ici, les matières sèches totales par unité de surface observées fluctuent

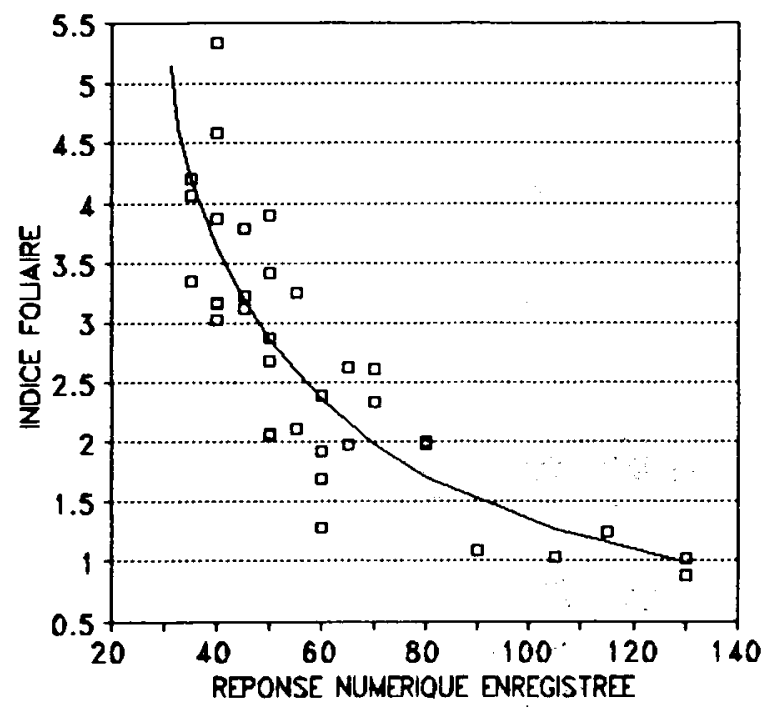

Fig 2. Liaison entre les indices foliaires des 36 prélèvements du dispositif et les réponses numériques correspondantes sur la photographie numérisée. Mission du 25 juin 1990.

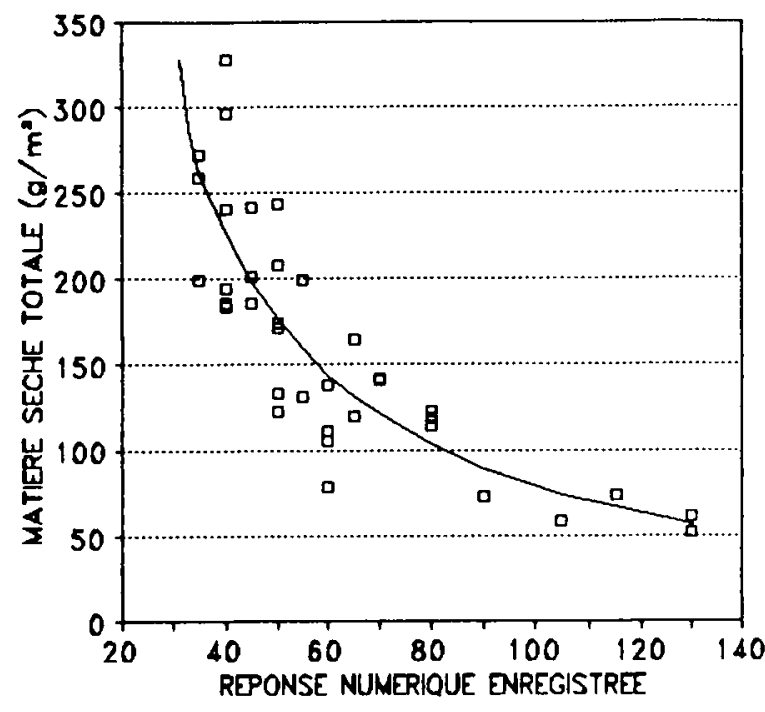

Fig 3. Liaison entre les matières sèches totales des 36 prélèvements du dispositif et les réponses numériques correspondantes sur la photographie numérisée. Mission du 25 juin 1990.

de 50 à $330 \mathrm{~g} / \mathrm{m}^{2}$ ) et la saturation de la réponse apparaît vers $300 \mathrm{~g} / \mathrm{m}^{2}$.

\section{Lame d'eau apportée}

Chez le soja, l'accroissement de la satisfaction de besoins en eau retarde l'apparition des processus de sénescence (Kadhem et al, 1985), donc l'augmentation de la réflectance dans le rouge. En utilisant ce principe, on peut établir lors de la sénescence, une relation entre la quantité d'eau apportée durant les irrigations (mesurée au moyen des pluviomètres d'appoint) et la réponse enregistrée sur les images numériques à l'issue des irrigations. Ceci n'est valable qu'à condition de travailler sur une zone où les réserves en eau du sol sont homogènes (ce qui est le cas de nos 4 parcelles comme l'a montré l'homogénéité de la levée dans cette partie du dispositif). La figure 4 montre le résultat obtenu sur la parcelle $\mathrm{n}^{\circ} 3$ à partir de la mission du 27 août 1990. La gamme des hauteurs d'eau observées (comprises entre 0 et $250 \mathrm{~mm}$ ) traduit le gradient des apports consécutif à l'utilisation d'un seul asperseur. La saturation des réponses numériques pour les fortes hauteurs d'eau indique des indices foliaires $>4,0$. La relation observée entre la réponse enregistrée et la hauteur d'eau suit une courbe en «S". Compte tenu toutefois de la précision recherchée lors de cette première approche expérimentale, il a été décidé 


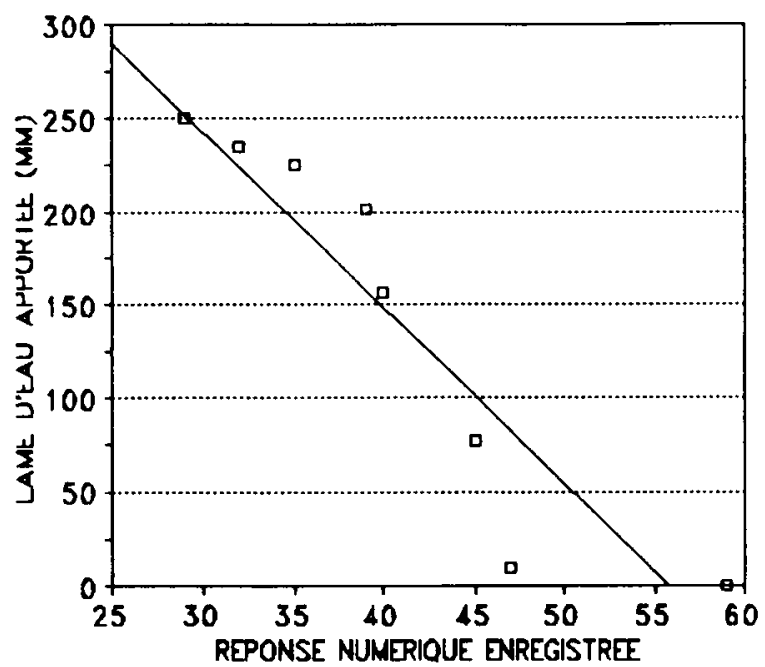

Fig 4. Liaison entre la lame d'eau apportée du 26 juin 1990 au 26 août 1990 sur les 8 points de mesure de la parcelle $n^{\circ}$ 3 et les réponses numériques correspondantes sur la photographie numérisée. Mission du 27 août 1990.

d'ajuster ce nuage de points par une droite. La qualité de la liaison observée $\left(r^{2}=0,87\right)$ montre que le facteur hydrique peut être correctement appréhendé au moyen du domaine visible et proche infrarouge, par l'intermédiaire des effets de l'eau sur la végétation (ici le taux de sénescence à la date de la mission).

Les relations statistiques observées dans nos conditions expérimentales montrent que l'imagerie visible et proche infrarouge permet l'appréhension des problèmes d'alimentation hydrique des plantes. À partir de ces relations, on peut utiliser le caractère synoptique des images visible et proche infrarouge pour extrapoler les relations obtenues à l'ensemble du dispositif expérimental observé.

\section{Établissement de cartes agronomiques thématiques}

Pour une image numérique donnée, si une liaison statistique est établie entre un descripteur de terrain et les valeurs numériques des pixels correspondants, on peut tenter, pour tous les autres pixels de l'image, de retrouver les valeurs de ce descripteur de terrain à partir des valeurs numériques observées. Ceci revient à extrapoler à toute une population une liaison statistique observée sur un échantillon. Pour cela, il faut que la liaison utilisée soit didentique pour l'ensemble des pixels de l'image. Nos conditions de travail satisfont à cette exigence car :
- les conditions d'acquisition des réponses numériques sont très proches pour tous les pixels utilisés du fait de la surface réduite utilisée sur les photographies;

- le sol présente une réponse spectrale stable sur l'ensemble du dispositif et il n'est utilisé qu'un seul cultivar;

- dans le cas où le descripteur est la lame d'eau, l'utilisation de l'irrigation comme seul facteur de différenciation permet d'éviter les confusions avec d'autres facteurs d'intensification (nutrition minérale par exemple).

En outre, dans chaque cas l'échantillon a été choisi pour présenter la plus large gamme possible de valeurs de descripteur de terrain, de façon à ce que l'ajustement réalisé couvre le mieux possible la totalité des cas de figure présents. Dans ces conditions, nous avons extrapolé les relations établies sur les pixels des échantillons (figs 2, 3 et 4) à l'ensemble des pixels des images correspondantes. Pour cela, les valeurs numériques initiales de tous les pixels de l'image sont converties au moyen des équations de passage entre réponse numérique sur l'image et valeur des descripteurs de terrain. Les nouvelles valeurs numériques obtenues correspondent, selon l'équation de passage choisie, à des valeurs d'indice foliaire, de matière sèche totale ou de hauteur d'eau apportée et les images ainsi recalculées sont des cartes numériques thématiques. Deux exemples en sont présentés : la carte des indices foliaires du 25 juin 1990 et la carte des hauteurs d'eau du 26 juin 1990 au 26 août 1990.

\section{Carte des indices foliaires}

Elle est obtenue à partir de l'équation de la figure 2. Elle permet de visualiser et d'analyser dans l'espace l'hétérogénéité du développement de la végétation, avant la mise en place des irrigations (fig 5). Ainsi, l'indice foliaire moyen calculé sur tout le dispositif (moyenne des 105364 pixels) est de 2,7. Cependant, par ordre d'indices foliaires croissants, on individualise 4 zones en raison de la variabilité spatiale des conditions édaphiques de croissance :

- zone 1, au centre et en bas de la parcelle (12 936 pixels, $398 \mathrm{~m}^{2}$, indice foliaire moyen = $1,6)$ : le faible développement observé sur cette zone est lié à une nature de sol plus sableuse ayant pénalisé les premiers stades de croissance par suite d'une moins bonne alimentation en eau des plantes; 
- zone 2, à droite de la parcelle (8 136 pixels, $251 \mathrm{~m}^{2}$, indice foliaire moyen $\left.=1,9\right)$ : la faible profondeur du sol est à l'origine des faibles valeurs d'indice foliaire.

- zone 3, au centre et en haut de la parcelle (11960 pixels, $368 \mathrm{~m}^{2}$, indice foliaire moyen = $2,4)$ : quoique moins marquée que dans la zone 1 , la texture plus sableuse du sol est ici aussi responsable du moindre développement des plantes;

- zone 4, tout le reste de la parcelle $(72332$ pixels, $2228 \mathrm{~m}^{2}$, indice foliaire moyen $=3,1$ ) : la profondeur croissante du sol, de la gauche vers la droite de la parcelle, explique l'accroissement des valeurs d'indice foliaire.

\section{Carte des apports d'eau}

Elle est réalisée pour la parcelle $\mathrm{n}^{\circ} 3$ à partir de l'équation de la figure 4 . Cette carte permet de visualiser le gradient de répartition de l'eau apportée pendant la période des irrigations (fig 6) et d'en quantifier l'influence sur la hauteur d'eau moyenne apportée sur la parcelle : seulement $160 \mathrm{~mm}$. Du fait que l'irrigation est dans ce cas le seul facteur d'intensification mis en place, on peut directement quantifier ce paramètre sans risque de confusion. Dans le cas où plusieurs facteurs interviennent, des risques de confusion apparaissent, et il faudra se limiter alors à une carte des biomasses ou des indices foliaires. Mais dans les 2 situations, cet outil est un moyen de contrôle spatialisé de l'homogénéité des conditions de croissance et de l'intensité des traitements mis en place sur un dispositif expérimental ou une parcelle agricole.

On note toutefois en haut à droite de la carte (zone entourée en violet sur la figure 6), un problème de confusion du signal : les lames d'eau calculées sont surestimées en raison de la présence d'adventices à forte absorption dans le rouge.

\section{Utilisation d'une carte comme outil spatial d'aide à la décision}

L'exemple suivant montre l'intérêt d'une description spatialisée de l'état du peuplement végétal dans l'optique d'une optimisation de la conduite de l'irrigation.

En raison du rôle assimilateur des feuilles, l'indice foliaire est utilisé dans de nombreux mo- dèles photosynthétiques pour déterminer l'accroissement potentiel de biomasse durant un laps de temps donné (Wiegand et Richardson, 1984). Étendu jusqu'à la récolte, ce raisonnement autorise une prévision de rendement si l'évolution des conditions pédoclimatiques (déterminant dans quelle mesure le potentiel d'élaboration de biomasse pourra être exploité) est précisée. Suivant ce principe, l'indice foliaire peut servir d'alarme pour l'irrigation, compte tenu d'un objectif visé de rendement. Or, dans le cadre de la conduite culturale, ce principe est appliqué sur la base d'une estimation moyenne d'indice foliaire par parcelle reposant sur un nombre limité de prélèvements. Comme l'a montré la figure 5 , cette hypothèse d'homogénéité est éloignée de la réalité et l'exemple suivant montre les conséquences d'une telle approximation.

A partir de la carte des indices foliaires du 25 juin 1990, il nous est possible de simuler une représentation plus ou moins sommaire de la répartition réelle des indices foliaires, correspondant par exemple à des protocoles de prélèvement plus ou moins lourds (fig 7 ) :

- protocole de prélèvement léger ne délivrant qu'une seule estimation moyenne pour l'ensemble du dispositif : 2,7;

- protocole de prélèvement plus lourd, délivrant une estimation moyenne d'indice foliaire pour chacun des blocs : $1,9,2,6,2,2,3,3$ et 3,5 pour les blocs 1-5;

- répartition détaillée des indices foliaires sur l'ensemble du dispositif.

La figure 8, détaille les conséquences de ces approximations plus ou moins poussées sur les surfaces à arroser, pour 3 indices foliaires seuils de déclenchement : $2,0,2,5$ et 3,0 . D'après la carte détaillée des indices foliaires (fig 8 , en bas), les surfaces nécessitant réellement une irrigation pour ces seuils (correspondant à des indices foliaires inférieurs à ces seuils et visualisés en bleu) représentent respectivement $20 \%, 29 \%$ et $42 \%$ de la parcelle. Lorsque I'on ne dispose que d'une valeur moyenne par bloc ou pour la parcelle (fig 8 au milieu et en haut), on est amené à :

- sous-estimer la surface nécessitant réellement intervention (lignes du haut et du milieu, indice foliaire seuil $=2,0$; ligne du haut, indice foliaire seuil = 2,5);

- surestimer la surface nécessitant réellement intervention (ligne du milieu indice foliaire seuil = 2,5; lignes du haut et du milieu, indice foliaire seuil $=3,0$ ). 


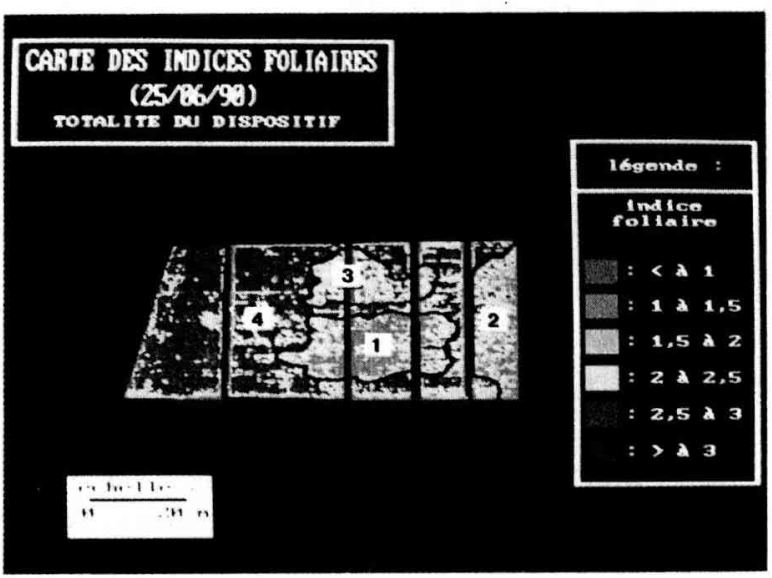

Fig 5. Carte des indices foliaires obtenue à partir de la photographie du 25 juin 1990 sur la totalité du dispositif (pour explications voir texte).

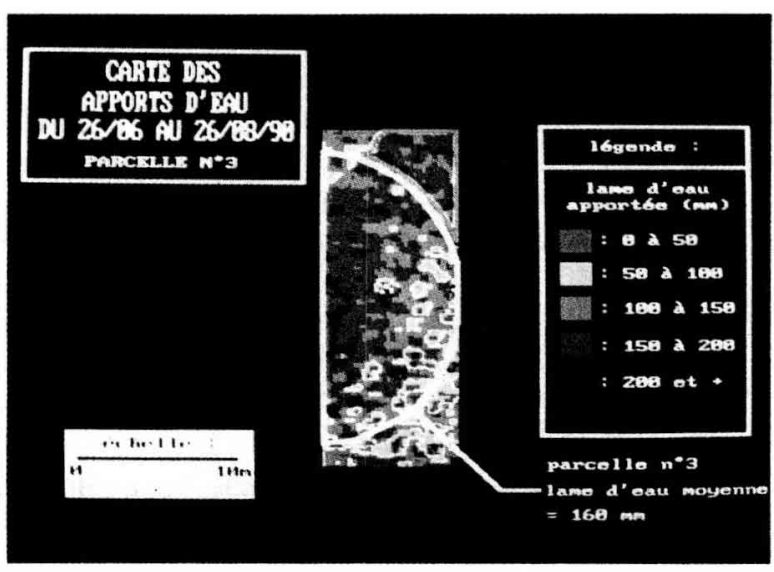

Fig 6. Carte des apports d'eau obtenue à partir de la photographie du 27 août 1990 sur la parcelle $n^{\circ} 3$ (pur explications voir texte).

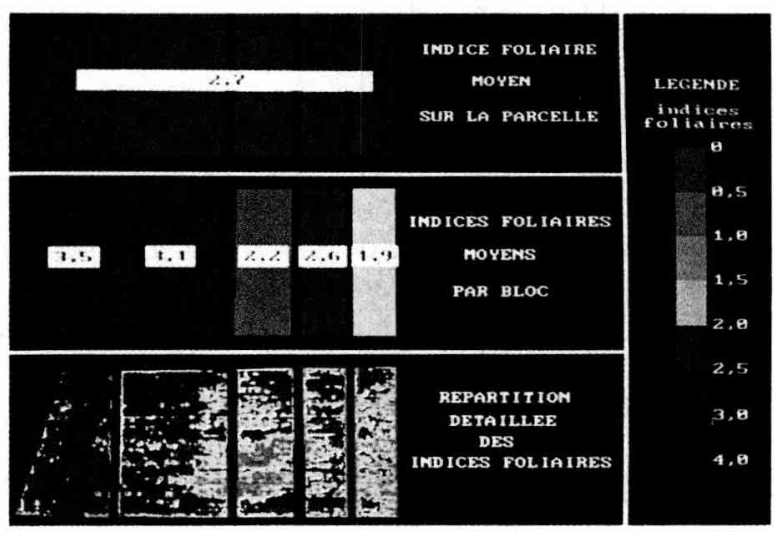

Fig 7. Indice foliaire moyen du dispositif (ligne du haut), indices foliaires moyens par bloc (ligne du milieu) et répartition détaillée des indices foliaires (ligne du bas) calculés à partir de la photographie du 25 juin. 1990.

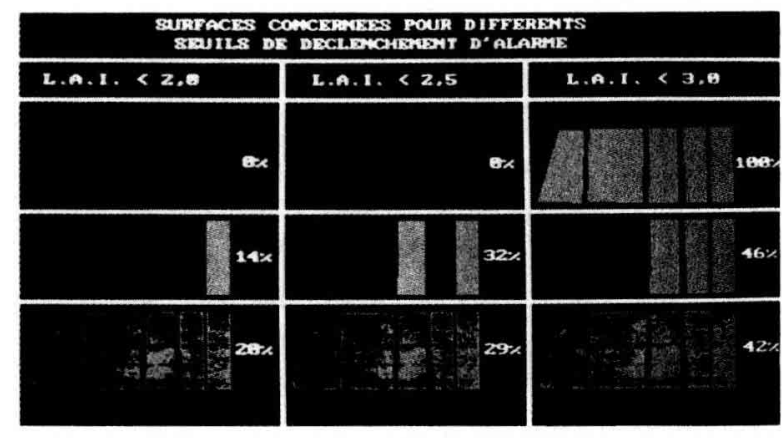

Fig 8. Conséquences d'une estimation plus ou moins sommaire de l'indice foliaire sur la surface de la parcelle à irriguer (en \% de la surface totale), pour trois indices foliaires de déclenchement de l'irrigation : 2,0,2,5 et 3,0 (surfaces nécessitant une irrigation en bleu). Mission du 25 juin 1990.

On note que l'erreur est d'autant plus grande que la représentation de la réalité est sommaire. De plus, l'utilisation de l'indice foliaire moyen (par bloc ou par parcelle) ne permet pas de connaître l'emplacement des points nécessitant l'intervention. Elle peut donc amener, malgré une estimation correcte de la surface à traiter, à intervenir sur des points n'en ayant pas le besoin (gaspillage de l'eau), ou inversement, à ne pas intervenir sur des points en ayant le besoin (objectif de rendement pas atteint).

\section{DISCUSSION ET CONCLUSION}

L'utilisation de la photographie aérienne IRC numérisée constitue une approche de l'état hydrique du peuplement végétal qui tient compte de l'hétérogénéité du couvert à l'échelle de la parcelle. Cette hétérogénéité est décrite de façon exhaustive sur des parcelles expérimentales plus ou moins intensifiées, au moyen de cartes numériques d'indice foliaire, de matière sèche totale, ou de hauteur d'eau apportée. Avant la mise en place de protocoles d'intensification, ces cartes permettent la prise en compte de l'hétérogénéité des sols. Après irrigation, ils permettent de contrôler l'intensité des traitements de la qualité de la répartition de l'eau. Enfin, ils débouchent sur une optimisation de la conduite de l'irrigation tenant compte de l'anisotropie du milieu, quoique l'application d'un tel résultat reste subordonnée à l'utilisation de techniques permettant d'opérer des apports différenciés dans l'espace.

La quantification directe d'un paramètre de traitement à partir de la réponse spectrale pose cependant un problème : la réponse spectrale n'est pas directement déterminée par la lame 
d'eau, mais par ses effets sur la croissance de la végétation. Deux facteurs de différenciation (alimentation hydrique et nutrition minérale par exemple) pouvant avoir des effets similaires sur la biomasse, l'origine d'une différenciation est délicate à préciser à partir de la seule réponse spectrale. Sur ce plan, la fiabilité du document présenté est assurée par l'homogénéité du sol sur cette partie du dispositif et par l'utilisation d'un seul facteur de différenciation : l'eau.

Dans le cadre de ce travail, les relations utilisées, de type statistique, ne peuvent être extrapolées en dehors du contexte pédo-climatique de l'étude, ainsi qu'à des conditions différentes d'acquisition des données spectrales. La généralisation des résultats nécessite dans un premier temps I'utilisation de données spectrales calibrées en valeurs de réflectance. Dans un deuxième temps, les valeurs de réflectance doivent être converties en descripteurs de l'état du couvert par l'inversion de modèles de détermination de la réponse des couverts (Guyot et Seguin, 1988). En outre, l'amélioration des résultats par l'utilisation d'indices de végétation doit être envisagée et la fiabilité des cartes thématiques doit être testée au moyen de prélèvements de contrôle. Enfin, les risques de confusion entre différents facteurs d'intensification nécessitent dans un premier temps l'application de cette méthode à des cas relativement simples ou clairement identifiés, ce qui est possible dans le cas des systèmes expérimentaux étudiés.

Sur la base des résultats obtenus, des développements intéressants apparaissent, actuellement mis en œuvre au laboratoire. Ils montrent que cette approche constitue une voie complémentaire d'étude du fonctionnement du peuplement végétal sur un dispositif expérimental d'une dizaine d'ha. Le caractère peu destructif de l'étalonnage des réponses numériques par rapport aux descripteurs de terrain (1-2\% de la surface observée) permet de répéter le diagnostic de l'état du peuplement végétal au cours du cycle cultural. Ainsi, la combinaison de cartes multidates permet l'analyse spatiale dynamique du fonctionnement agronomique du peuplement végétal. Les premiers résultats montrent en particulier que I'on accède à la mise en place des composantes du rendement. Ultérieurement, on peut étudier l'influence de différentes zones homogènes sur le développement moyen de la parcelle puis envisager la mise en œuvre d'une conduite culturale adaptée à cette variabilité spatiale.

\section{RÉFÉRENCES}

Curran PJ (1980) Relative reflectance data from preprocessed multispectral photography. Int $J$ Remote Sensing 1, 77-83

Curran PJ (1985) Aerial photography for the assessment of crop condition: a review. Applied Geography $5,347-360$

Everitt JH, Hussey MA, Escobar DE, Nixon PR, Pinkerton B (1986) Assessment of grassland phytomass with airborne video imagery. Remote Sensing of Environment 20, 299-306

Guyot G, Seguin B (1988) Possibilités d'utilisation de la télédétection satellitaire en agrométéorologie. Agronomie 8, 1-13

Holben BN, Tucker CJ, Cheng-Jeng Fan (1980) Spectral assessment of soybean leaf area and leaf biomass. Photogrammetric Engineering and Remote Sensing 46, 651-656

Kadhem FA, Specht JE, Williams JH (1985) Soybean irrigation serially timed during stages R1 to R6. I. Agronomic responses. Agron J 77, 291-298

Kollenkark JC, Daughtry CST, Bauer ME, Housley TL (1982) Effects of cultural practices on agronomic and reflectance characteristics of soybean canopies. Agron J 74, 751-758

Long DS, Nielsen GA, Carlson GR (1989) Use of aerial photographs for improving layout of field research plots. Appl Agric Res 4, 96-100

Marty JR, Cabelguenne M, Puech J, Hilaire A (1984) Perspectives d'optimisation technico-économique d'assolements de cultures à graines : céréales et oléoprotéagineux. Application à des sols argileux (terreforts) et limoneux (boulbènes) du Sud-Ouest de la France. INRA, Versailles

Mingeau M (1974) Étude de la sensibilité du soja à la sécheresse. Inf Tech CETIOM 47, 1-14

Tucker CJ, Elgin JH, McMurtrey JE (1979) Temporal spectral measurements of corn and soybean crops. Photogr Eng Remote Sens 45, 643-653

Wiegand CL, Richardson AJ (1984) Leaf area, light interception, and yield estimates from spectral components analysis. Agron J 76, 543-548 